\title{
Combined first-order reversal curve and x-ray microscopy investigation of magnetization reversal mechanisms in hexagonal antidot lattices
}

\author{
Joachim Gräfe, ${ }^{*}$ Markus Weigand, Claudia Stahl, Nick Träger, Michael Kopp, Gisela Schütz, and Eberhard J. Goering ${ }^{\dagger}$ \\ Max Planck Institute for Intelligent Systems, Stuttgart, Germany \\ Felix Haering, Paul Ziemann, and Ulf Wiedwald \\ Institute of Solid State Physics, Ulm University, Ulm, Germany \\ (Received 16 September 2015; revised manuscript received 1 November 2015; published 7 January 2016)
}

\begin{abstract}
The magnetization reversal in nanoscaled antidot lattices is widely investigated to understand the tunability of the magnetic anisotropy and the coercive field through nanostructuring of thin films. By investigating highly ordered focused ion beam milled antidot lattices with a combination of first-order reversal curves and magnetic x-ray microscopy, we fully elucidate the magnetization reversal along the distinct orientations of a hexagonal antidot lattice. This combination proves especially powerful as all partial steps of this complex magnetization reversal can be identified and subsequently imaged. Through this approach we discovered several additional steps that were neglected in previous studies. Furthermore, by imaging the microscopic magnetization state during each reversal step, we were able to link the coercive and interaction fields determined by the first-order reversal curve method to true microscopic magnetization configurations and determine their origin.
\end{abstract}

DOI: 10.1103/PhysRevB.93.014406

\section{INTRODUCTION}

Nanoscaled antidot lattices, i.e., a periodic arrangement of holes in a thin film, have been investigated within a broad scientific scope and in various host materials. In magnetic materials these nanostructures lead to the observation of interesting phenomena such as artificial spin ice [1-3] and spin glass [4], magnetic monopoles [5], and they can act as magnonic crystals that can be used as spin wave filters and spin wave guides [6-8]. In the context of data storage, antidot lattices are discussed as a type of bit patterned media that can overcome the superparamagnetic limit as there are no isolated magnetic islands [9-11]. Additionally, antidot lattices influence the magnetic properties of the host materials and can be used to tune the magnetic anisotropy [12-14] and coercivity [15-17].

In order to engineer the magnetic anisotropy and coercivity by using antidot lattices, it is of great importance to understand the microscopic origin and processes that lead to the change in magnetic properties. In the past, several approaches have been pursued to achieve this. Micromagnetic simulation found widespread use and indicated complex intermediate magnetization patterns during magnetization reversal [18-21]. On the other hand, microscopic investigations have been conducted by others using magneto-optical Kerr effect (MOKE) measurements [22] and photoelectron emission microscopy

\footnotetext{
*graefe@is.mpg.de

${ }^{\dagger}$ goering@is.mpg.de

${ }^{\ddagger}$ Present address: Faculty of Physics, University of Duisburg-Essen, Duisburg, Germany.
}

Published by the American Physical Society under the terms of the Creative Commons Attribution 3.0 License. Further distribution of this work must maintain attribution to the author(s) and the published article's title, journal citation, and DOI.
(PEEM) [16,23] using $\mathrm{x}$-ray magnetic circular dichroism (XMCD) as contrast mechanism. These studies also indicate the complex intermediate magnetization states, but are limited in spatial resolution [22] or the imperfect lattice order in self-organized antidot lattices [23]. Another approach to elucidating the reversal mechanisms are first-order reversal curves (FORC) $[18,21,24]$. However, these studies suffer from limited magnetic field resolution and the disorder in selforganized antidot lattices and, therefore, individual reversal processes have not been resolved so far.

FORC measurements promise to cover all microscopic magnetization states of a sample system without the need for the lateral resolution to spatially separate them [25]. Instead of spatially confining the measurement, this separation is achieved by the acquisition of multiple minor loops, starting at a multitude of different reversal fields $H_{r}$. The magnetization reversal is split into multiple FORC density peaks with different coercivities $H_{c}$ and interaction fields $H_{u}$ that might be interpreted as hysterons $[18,25,26]$. FORC measurements are increasingly used to investigate such problems in the context of geomagnetism [27-29], thin-film magnetism [30], and magnetic nanostructures [18]. Unfortunately, a direct interpretation of the FORC distribution as physically meaningful Preisach distribution is not always possible as the mathematical preconditions for a direct matching of both are often not met [25,31-33]. Therefore, the reliable interpretation of the physical meaning of an experimentally acquired FORC distribution is a major challenge [18,33,34].

Here, we present results from highly ordered nanoscaled antidot lattices that remove the influences from lattice defects or orientation mismatches. Using fast FORC measurements with high field resolution, previously undistinguished individual magnetization reversal processes are identified and their angular dependencies are shown. The unique combination of fast FORC measurements with scanning $\mathrm{x}$-ray microscopy (SXM) imaging with XMCD contrast allows identifying all microscopic steps of the complex microscopic magnetization 
reversal process. Matching all FORC density peaks to microscopic processes allows a full understanding of the FORC density and the magnetization reversal. Furthermore, linking the FORC density peaks to actual microscopic reversal events allows the discussion of the meaning of the coercivity and interaction field associated with a FORC density peak in nanostructured samples.

\section{METHODS}

The Fe thin films with a thickness of $20 \mathrm{~nm}$ for MOKE and SXM measurements were deposited by ion beam sputtering on $\mathrm{SiO}_{2}(300 \mathrm{~nm}) / \mathrm{Si}(001)$ and $\mathrm{Si}_{3} \mathrm{~N}_{4}(500 \mathrm{~nm}$, membranes)/Si(001), respectively, and capped by $2 \mathrm{~nm}$ of Al. MOKE measurements with $1^{\circ}$ angular resolution were performed to check that the iron thin films on both substrates had the same magnetic properties. Subsequently, the antidot pattern was etched by a focused $\mathrm{Ga}^{+}$ion beam in a FEI Nova 600 NanoLab DualBeam with a Raith ELPHY Multibeam pattern generator [35]. MOKE-based FORC measurements were conducted with a Durham Magneto Optics NanoMOKE3 equipped with an air-cooled vector electromagnet, capable of generating in-plane fields up to $120 \mathrm{mT}$. In longitudinal geometry, the laser beam was focused with an aspheric lens $(f=11 \mathrm{~mm})$ at an incidence angle of $45^{\circ}$ onto the sample, resulting in an imaging resolution of $1 \mu \mathrm{m}$ and an area of smaller than $5 \times 5 \mu \mathrm{m}^{2}$ that contributes to the magnetic signal. For each FORC measurement, 600 individual minor loops were acquired, each averaged 10 times at a cycling rate of $0.35 \mathrm{~Hz}$, thus, resulting in a total of 360000 data points. This means that, here, the FORC density is both influenced by the amount of magnetization change and the degree of irreversibility of the process leading to this magnetization change, i.e., the probability of a stochastic magnetization reversal process being irreversible affects the FORC density. FORC density calculations from the acquired minor loops were done using customized MATLAB code. This was numerically achieved by fitting $[29,36]$ a part of the $M\left(H, H_{r}\right)$ data to

$M\left(H, H_{r}\right)=a_{1}+a_{2} H+a_{3} H^{2}+a_{4} H_{r}+a_{5} H_{r}^{2}-2 a_{6} H_{r} H$

and extracting the parameter $a_{6}$ as FORC density $\rho$ :

$$
\rho\left(H, H_{r}\right)=-\frac{1}{2} \frac{\partial^{2} M\left(H, H_{r}\right)}{\partial H \partial H_{r}}=a_{6} .
$$

Subsequently, the measurement axes $H$ and $H_{r}$ were transformed into $H_{c}$ and $H_{u}$ axes according to

$$
H_{u}=\frac{1}{2}\left(H+H_{r}\right)
$$

and

$$
H_{c}=\frac{1}{2}\left(H-H_{r}\right) .
$$

After smoothing, this processing scheme leads to FORC distributions with a minimum resolution of $\geqslant 0.2 \mathrm{mT}$ and a baseline resolution of $\leqslant 4 \mathrm{mT}$. Further details about the acquisition scheme and the data evaluation can be found elsewhere [35,36].

SXM measurements were conducted at the MPI IS operated MAXYMUS end station at the UE46-PGM2 beam line at the BESSY II synchrotron radiation facility. The samples were illuminated under $30^{\circ}$ grazing incidence by circularly polarized light in an applied in-plane field of up to $240 \mathrm{mT}$ that was generated by a set of four rotatable permanent magnets [37]. The photon energy was set to the absorption maximum of the $\mathrm{Fe} L_{3}$ edge to get optimal XMCD contrast for imaging. The transmission through the holes, where there is no material and thus no x-ray absorption, was used as an internal intensity $I_{0}$ reference to normalize the measured intensities to the incident beam intensity. Intensities were locally averaged using a Gaussian filter in ImageJ [38]. Images taken at different photon helicities were registered using ImageJ with TurboReg $[35,39]$.

\section{RESULTS}

The general layout of a hexagonal antidot lattice, investigated here, is shown in Fig. 1(a). Hexagonal lattices feature two geometrically distinct directions: the nearest-neighbor (nn) direction which runs along a close-packed string of holes and shows the smallest distance between two neighboring antidots; and the next-nearest neighbor (nnn) direction which shows the largest distance between two neighboring antidots and has an angle of $30^{\circ}$ to the nn direction. Because of the hexagonal symmetry of the antidot lattice these directions are repeated every $60^{\circ}$. In magnetic antidot lattices where the hole spacing $a$ and diameter $d$ ratio is large enough to allow an uninterrupted material strip along the $n n$ direction, the nn directions are the in-plane easy and the nnn directions the hard axes of the system [14]. This is also reflected in the angular dependence of the coercivity $H_{c}$, shown in Fig. 1(b). While the unstructured Fe thin film features a small and isotropic coercivity, the antidot lattices shown here result in a steep increase of $H_{c}$ compared to the unstructured thin film and the sixfold symmetry of the antidot lattice is reproduced in the magnetic properties. Therefore, an in-plane easy and hard axis is found every $60^{\circ}$ with an enclosed angle of $30^{\circ}$ between the two. However, the $\mathrm{nn}$ and nnn directions not only differ in coercivity, but also in the magnetization reversal behavior, which is discussed in the following where we focus on an antidot lattice with spacing $a=400 \mathrm{~nm}$ and diameter $d=50 \mathrm{~nm}$ in a $20 \mathrm{~nm}$ Fe film.

\section{A. Magnetization reversal along nn directions}

The major loop and the FORC density for the magnetization reversal along the nn direction are shown in Figs. 2(a) and 2(b), respectively. The FORC density features two irreversible processes I $\left(H_{c}=2 \mathrm{mT}\right)$ and II $\left(H_{c}=13 \mathrm{mT}\right)$ that are also reflected in jumps in the major hysteresis loop. Both FORC density peaks show no shift along the interaction field $H_{u}$ axis. This is in line with a magnetization reversal by independent domain nucleation and propagation as proposed in previous studies $[14,18]$. The major part of the magnetization reversal is achieved by the irreversible process II, as indicated by the large FORC density at its position. Both processes exhibit a rather small peak width for their corresponding FORC density peaks, which indicates a narrow field range for domain propagation after their nucleation and, in general, the high quality of the samples. 


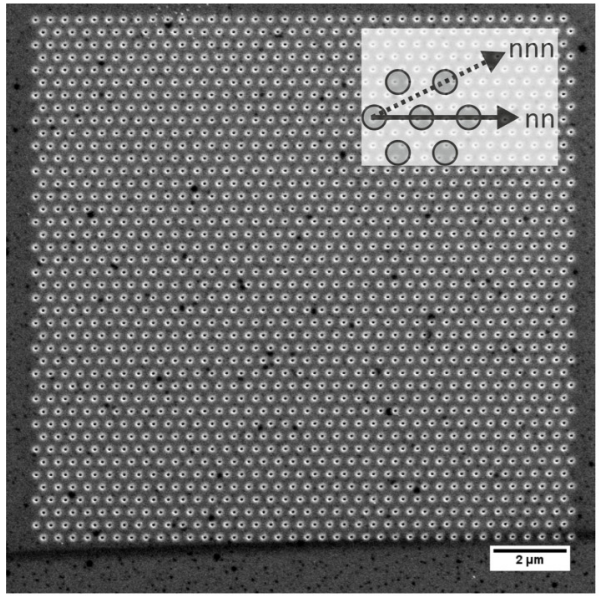

(a)

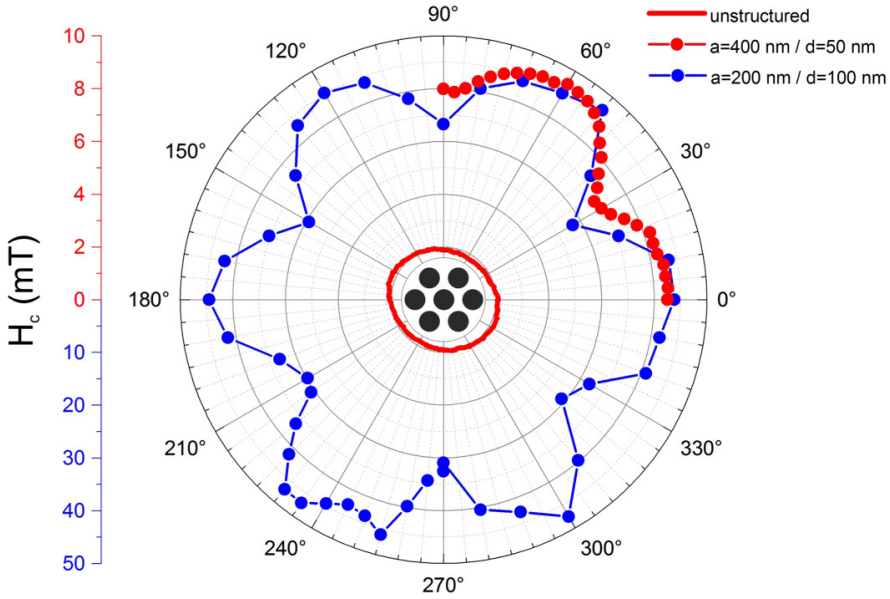

(b)

FIG. 1. General properties of hexagonal antidot lattices in $20 \mathrm{~nm}$ thick Fe films indicated by (a) a SEM image of an antidot lattice (antidot spacing $a=400 \mathrm{~nm}$ and diameter $d=50 \mathrm{~nm}$ ) and (b) the angular dependence of the coercivity in two antidot lattices with different spacing $a$ and diameter $d$ with a nn direction aligned along $0^{\circ}$. For comparison, the isotropic coercivity of the unstructured thin film is also shown.

The same magnetization reversal along the nn direction is shown as a series of several x-ray micrographs in Fig. 3. These reveal that the magnetization reversal actually occurs in three separable steps. Therefore, three types of domains with the magnetization aligned along the $60^{\circ}$ [Fig. 3(b)], $120^{\circ}$ [Fig. 3(c)], and $180^{\circ}$ [Fig. 3(d)] directions are observable. Hence, the majority of the magnetization is always oriented along an easy axis at $0^{\circ}, 60^{\circ}, 120^{\circ}$, or $180^{\circ}$. All three domain types propagate in a way that their domain walls are always aligned along a nn direction with its close-packed string of antidots, which is in line with previous findings [14]. Thus, the domain walls span the shortest distance between two adjacent holes, minimizing the domain-wall length and associated exchange energy. It is noteworthy that each type of domain propagates along a different $\mathrm{nn}$ direction: along the $60^{\circ}, 180^{\circ}$, and $120^{\circ}$ directions, respectively. This means that for the second and the third processes the magnetization within the domain is not aligned parallel to the domain wall and they are canted at an angle of $60^{\circ}$. Although some intermediate magnetization rotation occurs, there is always a nucleation of domains when the magnetization crosses an easy axis at a nn direction.

The first two domain nucleation and propagation processes can easily be linked to the irreversible processes I and II in the FORC density map. Although the final process is also a domain nucleation process, resulting in domains with their magnetization aligned along the $180^{\circ}$ direction, it does not manifest as peak in the FORC density. As the FORC distribution only indicates irreversible magnetization reversal steps, this suggests that process III is not irreversible. Indeed, we find in SXM measurements that process III is quasireversible, i.e., when reducing the external magnetic field the $180^{\circ}$ domains reversibly move back the same way they extended. While the majority of the reversal is achieved by irreversible process II that is linked to domain propagation along the external field direction and the effective rotation of the magnetization across the $90^{\circ}$ direction, we observe two additional processes that have not been described in literature $[16,18,23]$ so far, namely, processes I and III, highlighting the complexity of the magnetization reversal in antidot lattices.

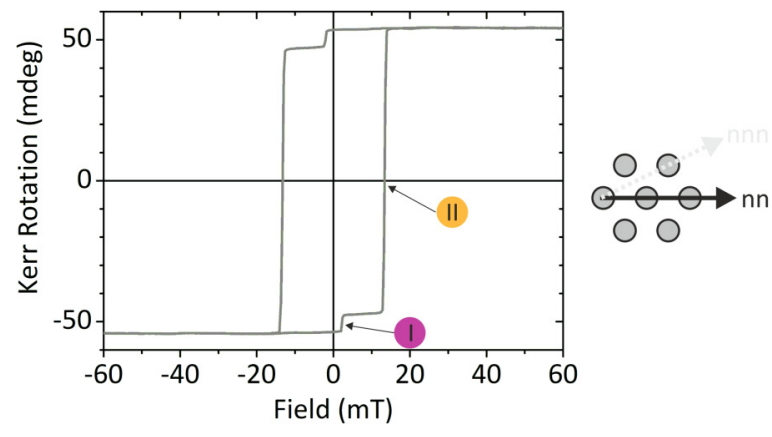

(a)

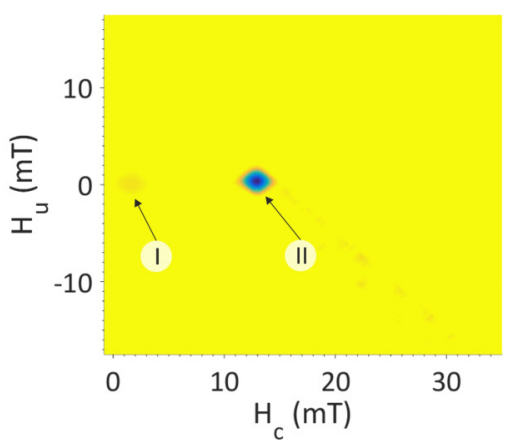

(b)

FIG. 2. MOKE measurements of a hexagonal antidot lattice (spacing $a=400 \mathrm{~nm}$ and diameter $d=50 \mathrm{~nm}$ ) in a $20 \mathrm{~nm}$ Fe film measured along the nn direction. Both the (a) major loop and (b) FORC density are shown. 


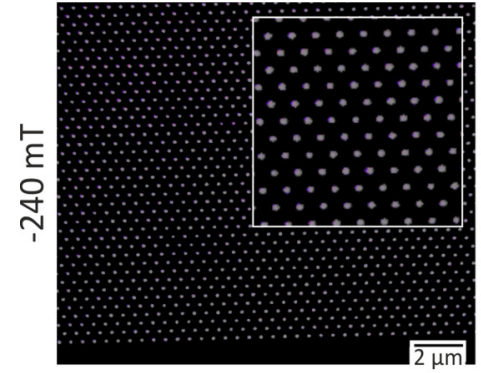

(a)

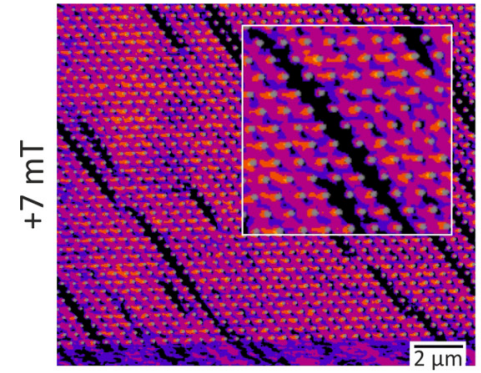

(b)
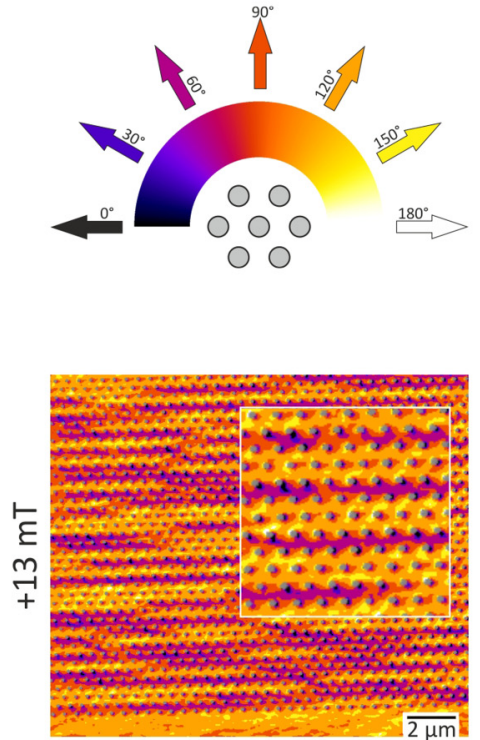

(c)

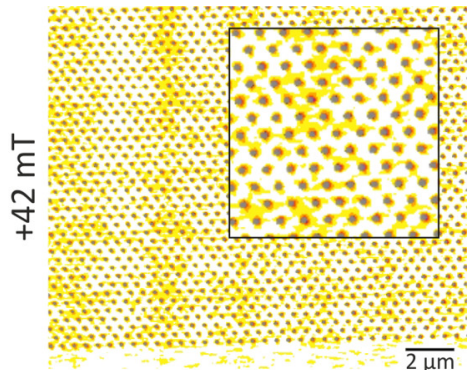

(e)

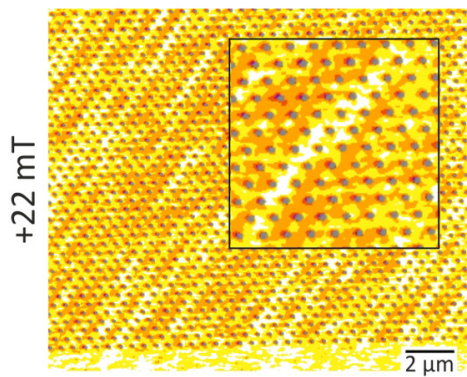

(d)

FIG. 3. SXM measurement series of a hexagonal antidot lattice (spacing $a=400 \mathrm{~nm}$ and diameter $d=50 \mathrm{~nm}$ ) in a $20 \mathrm{~nm}$ Fe film during magnetization reversal along the $\mathrm{nn}$ direction. Images at an applied external field of (a) $-240 \mathrm{mT}$ (negative saturation), (b) $7 \mathrm{mT}$, (c) $13 \mathrm{mT}$, (d) $22 \mathrm{mT}$, and (e) $42 \mathrm{mT}$ (positive saturation) are shown. Insets show a $2 \times$ magnified detail. The angular orientation of the local magnetization is indicated by the color code shown in the center. See Supplemental Material for a video of the full magnetization reversal including additional field steps [40].

\section{B. Magnetization reversal along nnn directions}

Figure 4(a) shows the major loop hysteresis of the magnetization reversal along the nnn direction of the antidot lattice. From this major loop hysteresis it is already obvious that the reversal mechanisms are much more complex than for the nn direction. This is also reflected in the FORC distribution, shown in Fig. 4(b), that shows more features than the one discussed previously for the nn direction. The FORC distribution shows two irreversible processes with large FORC density at $H_{c}=17 \mathrm{mT}, H_{u}=11 \mathrm{mT}$ and $H_{c}=9 \mathrm{mT}, H_{u}=0 \mathrm{mT}$ (marked II and III, respectively), that correspond to the largest part of the magnetization reversal. It is noteworthy that on an external field scale process II happens at a lower applied field than process III, i.e., $H_{\mathrm{ext}}=6 \mathrm{mT}$ and $H_{\text {ext }}=9 \mathrm{mT}$, respectively. The large negative interaction field $H_{u}$ leads to this process preceding the one with a formally smaller $H_{c}$ value. Additionally, there are two peaks at $H_{c}=1 \mathrm{mT}$ and $H_{c}=28 \mathrm{mT}$ without shift along the $H_{u}$ axis (marked I and IV, respectively) with lower FORC density. In the case of process I, this can be attributed to the smaller change in magnetization that is associated with this process. For process IV, a quasireversible domain-wall movement is found in SXM measurements, like before for the final step of the magnetization reversal along the nn direction. Furthermore, a so-called reversible ridge [29] is found for $H_{c}=0$, indicating that reversible rotation is also contributing to the overall magnetization reversal.

The increased complexity of magnetization reversal processes is also found in SXM measurements, shown in Fig. 5. The first process observed is the alignment of the magnetization along the $60^{\circ}$ direction. As the FORC distribution exhibits both a reversible ridge and a peak close to $H_{c}=0$ it is not unlikely that there is a partially irreversible formation of domains with a magnetization that rotates past the first easy axis at $30^{\circ}$ combined with a reversible rotation. An initial relaxation of the magnetization away from the $0^{\circ}$ direction at zero field is caused by a deflection of the magnetization at the hole edges which is favorable due to the reduction of stray fields into the antidot [14,35].

In process II domains with their magnetization oriented along the next easy axes at $90^{\circ}$ enter the antidot lattice and expand along the $\mathrm{nn}$ direction at $30^{\circ}$, shown in Figs. 5(c) and 5(d). After the domains nucleated and the magnetization overcame the nn direction, the local magnetization does not remain at $90^{\circ}$ but immediately rotates further, shown in Figs. 5(d) and 5(e). The SXM images clearly resolve that a part within the antidot lattice unit cell rotates towards the nnn direction at $120^{\circ}$. Thus, many small sub-domains are formed as bubbles between two antidots [cf. enlargement of Fig. 5(e)]. It is noteworthy that the magnetization in these subdomains points into globally hard axes, however, at a local scale this orientation allows the magnetization to avoid pointing into the hole. Thus, when only an area smaller than the unit cell is considered, the preferential magnetization orientation is parallel to the nnn direction. This highlights that the determination of the easy or hard axis from the major loop hysteresis alone may not be accurate on a microscopic scale. As this additional rotation further towards the applied field direction is only possible after the domains oriented along the $90^{\circ}$ direction have nucleated, this makes the initial nucleation 


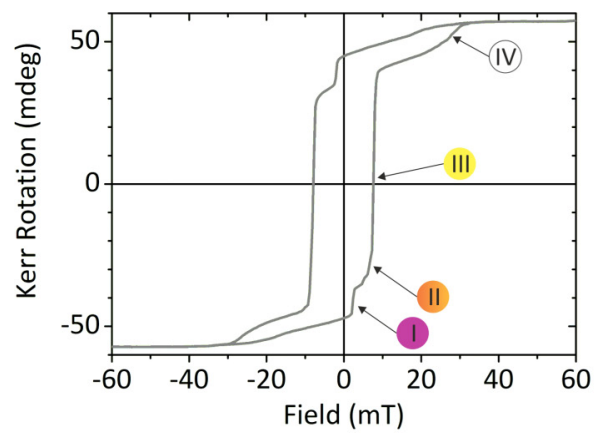

(a)

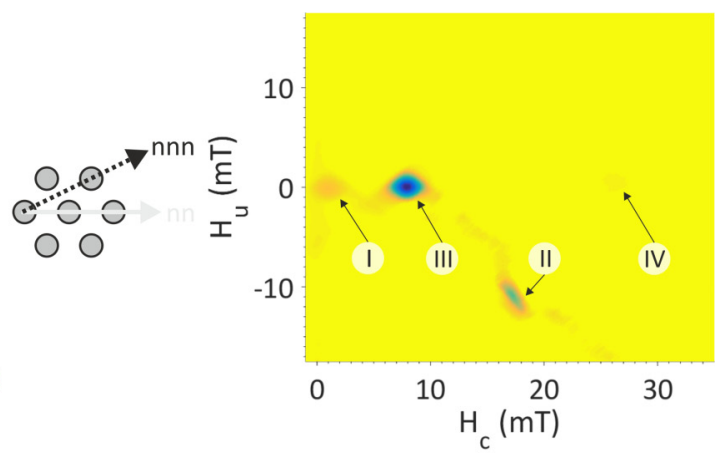

(b)

FIG. 4. MOKE measurements of a hexagonal antidot lattice (spacing $a=400 \mathrm{~nm}$ and diameter $d=50 \mathrm{~nm}$ ) in a $20 \mathrm{~nm}$ Fe film measured along the nnn direction. Both the (a) major loop and (b) FORC density are shown.

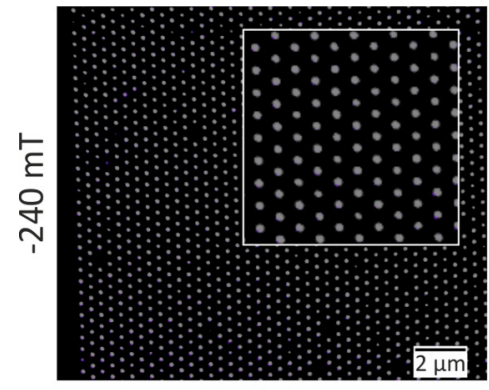

(a)

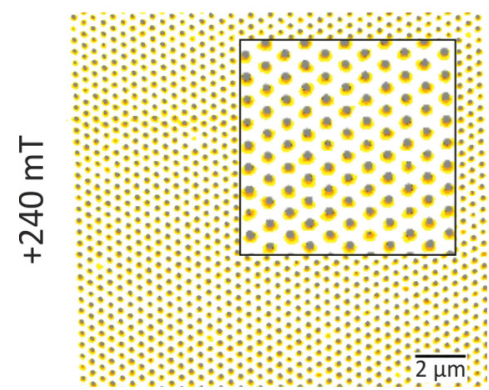

(h)

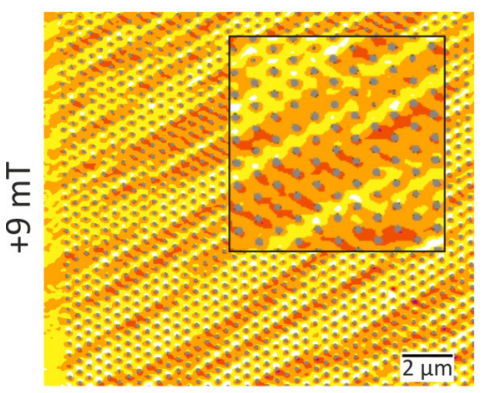

(g)

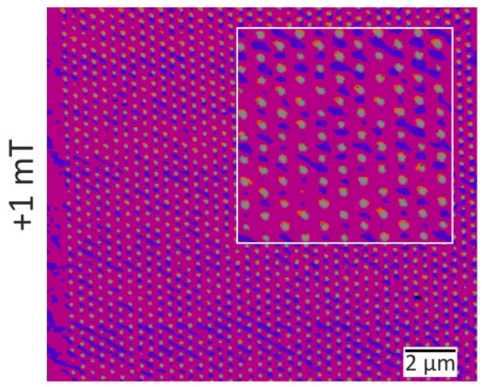

(b)
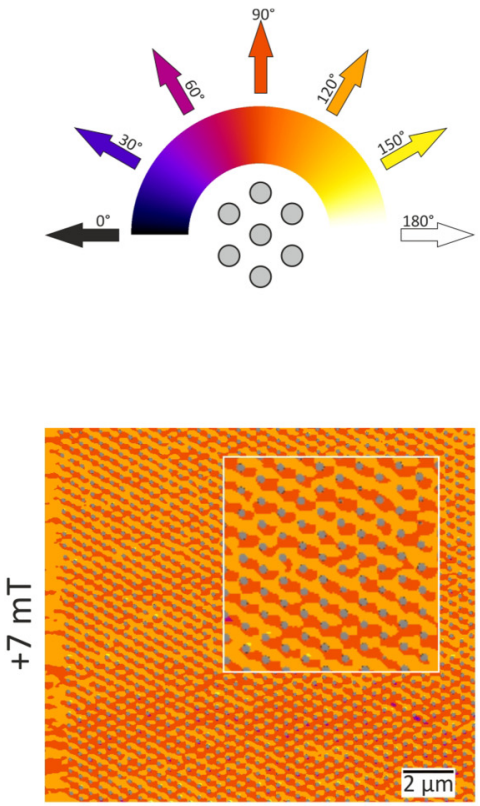

(f)

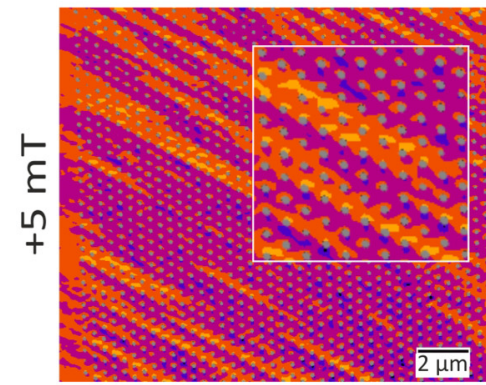

(d)

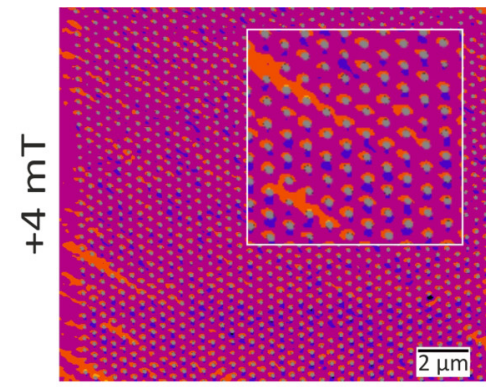

(c)

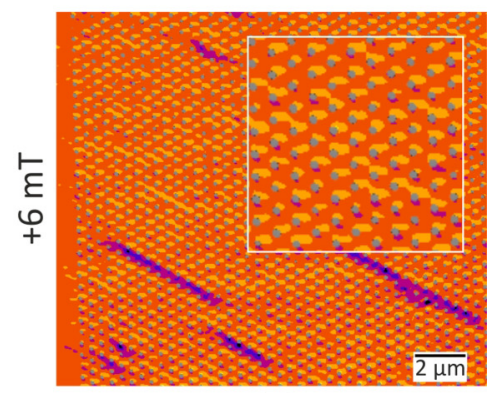

(e)

FIG. 5. SXM measurement series of a hexagonal antidot lattice (spacing $a=400 \mathrm{~nm}$ and diameter $d=50 \mathrm{~nm}$ ) in a $20 \mathrm{~nm}$ Fe film during magnetization reversal along the nnn direction. Images at an applied external field of (a) $-240 \mathrm{mT}$ (negative saturation), (b) $1 \mathrm{mT}$, (c) $4 \mathrm{mT}$, (d) $5 \mathrm{mT}$, (e) $6 \mathrm{mT}$, (f) $7 \mathrm{mT}$, (g) $9 \mathrm{mT}$, and (h) $240 \mathrm{mT}$ (positive saturation) are shown. Insets show a $2 \times$ magnified detail. The angular orientation of the local magnetization is indicated by the color code shown in the center. See Supplemental Material for a video of the full magnetization reversal including additional field steps [40]. 
of these domains energetically favorable. Therefore, providing a possible source for the interaction field $H_{u}$ that is exhibited by the corresponding peak in FORC density of process II.

Subsequently, at slightly larger applied fields $H_{\text {ext }}$, process III occurs, that is associated microscopically to the nucleation of domains with the magnetization aligned along the nn direction at $150^{\circ}$ and their propagation along that same direction as shown in Fig. $5(\mathrm{~g})$. The combination of processes II and III responsible for the majority of the magnetization reversal, spanning a magnetization rotation from $60^{\circ}$ to $150^{\circ}$, and are almost inseparable in the major loop hysteresis. Finally, the quasireversible process IV results in a rotation out of the easy axis into saturation and alignment of the magnetization at the $180^{\circ}$ nnn direction.

In summary, the magnetization reversal along the nnn direction occurs in two irreversible and two quasireversible processes, exhibiting a peak in the FORC distribution, and additional reversible rotation, both before process I and between the irreversible steps. The principal steps are based, very similar to the magnetization reversal along the nn direction, on the nucleation of different domain types whenever the magnetization crosses a nn direction. These domains only propagate along the $\mathrm{nn}$ direction while the magnetization is not necessarily aligned parallel to the domain wall. Each nucleation and propagation of domains is accompanied by an additional rotation of the magnetization to further align with the applied field.

\section{DISCUSSION}

While the magnetization reversal processes in nanoscaled antidot lattices are quite complex and feature overlapping fundamental reversal steps, there are still some common features for these processes. When the orientation of the magnetization crosses an easy axis, this results in the formation of domains. These domains propagate through the antidot lattice along the nn directions, so that the domain walls span the shortest distance between two adjacent holes, thus minimizing the exchange energy associated to that domain wall. However, the orientation of the magnetization is not necessarily parallel to the domain wall. As the formation of magnetic surface charges at the antidot edge would incur a significant energy penalty, the magnetization tends to point away from the holes. On the other hand, the positioning of the domain wall at the shortest stretch between two holes allows the minimization of the exchange energy associated with that domain wall. The combination of these two geometrical constraints favors a misalignment of the magnetization and the domain wall. After domain nucleation and propagation, the magnetization can rotate reversibly until crossing another nn direction would be necessary.

In linking FORC density peaks to microscopic processes, we find that these are typically associated to domain nucleation and propagation processes. As the FORC density represents irreversible switching events the peak intensity is not only determined by the magnetization change, but also the degree of irreversibility of that process. In highly ordered antidot lattices, the local domain-wall pinning strength is so well defined that both the domain-wall position is reproducible over several cycles as well as the domain-wall movement may be reversible to some degree. This quasireversible domain-wall movement leads to a low degree of irreversibility and, hence, a small FORC density for these processes.

During the magnetization reversal of a nanoscaled antidot lattice, the same magnetic moments take several intermediate states, thus, the premise of a bistable state of the Preisach distribution is not met by this system [31]. Furthermore, the wiping-out and congruency properties that are required to directly match the Preisach and FORC distributions may not be fulfilled here [32]. Hence, the nominal coercivity $H_{c}$ and the interaction field $H_{u}$ of the irreversible processes in the FORC distribution cannot be interpreted as classical coercive and interaction fields of a magnetic particle. What can be directly deduced from the FORC distribution is the existence of several irreversible process at given external fields $H_{\text {ext }}$ (with $H_{\text {ext }}=H_{c}+H_{u}$ ). The bistable state can be virtually achieved when only considering two states of the multistep reversal individually, e.g., only looking at the magnetization jump from $60^{\circ}$ to $120^{\circ}$ across a nn direction. $H_{c}$ is then an indicator for the energy required to achieve this specific magnetization change, but not necessarily a true coercive field. Therefore, a subsequent step may well have a lower $H_{c}$ in the FORC distribution as the additional energy required for this step may indeed be smaller. While $H_{c}$ is not a classical coercivity in the case of antidot lattices, it still provides valuable information about the domain-wall pinning strength of the antidots. Furthermore, the narrow distribution in $H_{c}$ clearly indicates that the pinning strength is identical in the whole lattice, due to the very uniform hole-size distribution. This would not be achievable with a self-organization based nanostructuring approach that typically results in a wider hole-size distribution and misalignment of structural domains.

The question of the physical interpretation of the FORC interaction field $H_{u}$ is widely discussed in literature as it cannot always be interpreted as a coupling field to the surrounding magnetization landscape $[33,34]$. For the antidot lattice magnetization reversal, only one peak in the FORC distribution exhibits an interaction field $H_{u}$, namely, process II for the reversal along the nnn direction. The distinction of this irreversible process is that it is microscopically coupled to a second process: the nucleation and propagation of domains allow an additional subsequent rotation of the magnetization. In light of the previous discussion of the origin of $H_{c}$, it can be argued that the possibility of a subsequent reversible rotation to further align with the external field makes the initial nucleation of the domains more favorable, thus providing an additional energy gain and a negative FORC $H_{u}$, shifting this process to lower external field values. After nucleation of these domains, the magnetization can rotate further; reversing the nucleation step would require rotation against the external field first, thus, there is an interaction between the two processes. Here, it is apparent that the FORC $H_{u}$ is not a simple dipole interaction field of neighboring magnetic moments that interact, but more generally an additional energy contribution.

Not only the interpretation of FORC density peaks as hysterons which is based on the assumption of a bistable Preisach-type process is more complicated in this system, but for the same reasons the determination of the easy and hard axes orientation is not straightforward. On a macroscopic scale, the nn direction is clearly the easy axis as its major 
loop hysteresis features both a larger magnetization at zero field as well as a larger field that is required to reach zero magnetization. However, on a microscopic scale between two intermediate magnetization states, this is not as straightforward and the preferred orientation of the magnetization then depends on both the magnetization history and the surrounding magnetization landscape.

\section{SUMMARY}

We have fully elucidated the angle-dependent magnetization reversal in hexagonal antidot lattices. The magnetization reversal along the nn direction occurs in two irreversible and one quasireversible domain-wall nucleation and propagation steps. The more complex magnetization reversal along the nnn direction occurs via a combination of two irreversible, two quasireversible, and one reversible step with different domain patterns. These processes were separated by FORC measurements with high field resolution and their microscopic magnetization state was imaged using SXM with XMCD contrast. Using highly ordered antidot lattices with uniform hole-size distribution allowed distinguishing these individual steps as the pinning strength distribution was narrow and there was no overlap of different processes due to angular misaligned structure domains of the antidot lattice.

Combining FORC that promises information about all microscopic magnetization states with $\mathrm{x}$-ray microscopy to image these states allowed to examine the origin of $H_{c}$ and $H_{u}$ fields determined from the FORC distribution. Although a strict correlation between the Preisach distribution and the FORC distribution is not observed, there is still valuable information to be gained from the FORC distribution. FORC density peaks need to be clearly distinguished from Preisach hysterons, thus, moving away from the classical view of coercive and interaction field and towards a more general picture of energies that are required for a specific magnetization change. Still, $H_{u}$ may provide information about additional interacting energy contributions to a reversal process, however, this must not be limited to the interaction of a magnetic moment with its surrounding magnetization landscape.

\section{ACKNOWLEDGMENTS}

The authors would like to thank M. Bechtel for support during beam times and B. Ludescher for thin-film deposition. Furthermore, we are grateful to U. Eigenthaler for performing excellent FIB structuring. Helmholtz Zentrum Berlin is acknowledged for allocating beam time at the BESSY II synchrotron radiation facility. Financial support by the BadenWürttemberg Stiftung in the framework of the Kompetenznetz Funktionelle Nanostrukturen is gratefully acknowledged.
[1] J. P. Morgan, A. Stein, S. Langridge, and C. H. Marrows, Nat. Phys. 7, 75 (2010).

[2] F. Haering, U. Wiedwald, T. Haberle, L. Han, A. Plettl, B. Koslowski, and P. Ziemann, Nanotechnology 24, 055305 (2013).

[3] L. J. Heyderman, Nat. Nanotechnol. 8, 705 (2013).

[4] M. F. Laguna, C. A. Balseiro, D. Domínguez, and F. Nori, Phys. Rev. B 64, 104505 (2001).

[5] E. Mengotti, L. J. Heyderman, A. F. Rodriguez, F. Nolting, R. V. Hugli, and H. B. Braun, Nat. Phys. 7, 68 (2011).

[6] M. J. Pechan, C. Yu, R. L. Compton, J. P. Park, and P. A. Crowell, J. Appl. Phys. 97, 10J903 (2005).

[7] B. Lenk, H. Ulrichs, F. Garbs, and M. Munzenberg, Phys. Rep. 507, 107 (2011).

[8] H. Yu, G. Duerr, R. Huber, M. Bahr, T. Schwarze, F. Brandl, and D. Grundler, Nat. Commun. 4, 2702 (2013).

[9] R. P. Cowburn, A. O. Adeyeye, and J. A. C. Bland, Appl. Phys. Lett. 70, 2309 (1997).

[10] L. Torres, L. Lopez-Diaz, and J. Iñiguez, Appl. Phys. Lett. 73, 3766 (1998).

[11] M. B. A. Jalil, J. Appl. Phys. 93, 7053 (2003).

[12] F. J. Castano, K. Nielsch, C. A. Ross, J. W. A. Robinson, and R. Krishnan, Appl. Phys. Lett. 85, 2872 (2004).

[13] C. C. Wang, A. O. Adeyeye, and N. Singh, Nanotechnology 17, 1629 (2006).

[14] F. Haering, U. Wiedwald, S. Nothelfer, B. Koslowski, P. Ziemann, L. Lechner, A. Wallucks, K. Lebecki, U. Nowak, J. Gräfe, E. Goering, and G. Schütz, Nanotechnology 24, 465709 (2013).
[15] C. C. Wang, A. O. Adeyeye, and Y. H. Wu, J. Appl. Phys. 94, 6644 (2003).

[16] L. J. Heyderman, F. Nolting, D. Backes, S. Czekaj, L. LopezDiaz, M. Klaui, U. Rudiger, C. A. F. Vaz, J. A. C. Bland, R. J. Matelon, U. G. Volkmann, and P. Fischer, Phys. Rev. B 73, 214429 (2006).

[17] G. Ctistis, E. Papaioannou, P. Patoka, J. Gutek, P. Fumagalli, and M. Giersig, Nano Lett. 9, 1 (2009).

[18] F. Béron, K. R. Pirota, V. Vega, V. M. Prida, A. Fernández, B. Hernando, and M. Knobel, New J. Phys. 13, 013035 (2011).

[19] B. Van de Wiele, A. Manzin, A. Vansteenkiste, O. Bottauscio, L. Dupré, and D. De Zutter, J. Appl. Phys. 111, 053915 (2012).

[20] P. Gawroński, K. J. Merazzo, O. Chubykalo-Fesenko, A. Asenjo, R. P. del Real, and M. Vázquez, Europhys. Lett. 100, 17007 (2012).

[21] S. Michea, J. L. Palma, R. Lavín, J. Briones, J. Escrig, J. C. Denardin, and R. L. Rodríguez-Suárez, J. Phys. D: Appl. Phys. 47, 335001 (2014).

[22] S. Mallick and S. Bedanta, J. Magn. Magn. Mater. 382, 158 (2015).

[23] K. J. Merazzo, C. Castan-Guerrero, J. Herrero-Albillos, F. Kronast, F. Bartolome, J. Bartolome, J. Sese, R. P. del Real, L. M. Garcia, and M. Vazquez, Phys. Rev. B 85, 184427 (2012).

[24] M. P. Proenca, K. J. Merazzo, L. G. Vivas, D. C. Leitao, C. T. Sousa, J. Ventura, J. P. Araujo, and M. Vazquez, Nanotechnology 24, 475703 (2013). 
[25] F. Béron, L.-P. Carignan, D. Ménard, and A. Yelon, Electrodeposited Nanowires and Their Applications, edited by N. Lupu (Intech, Croatia, 2010), Chap. 7.

[26] F. Béron, D. Ménard, and A. Yelon, J. Appl. Phys. 103, 07 D908 (2008).

[27] C. R. Pike, A. P. Roberts, and K. L. Verosub, J. Appl. Phys. 85, 6660 (1999).

[28] A. P. Roberts, C. R. Pike, and K. L. Verosub, J. Geophys. Res.: Solid Earth 105, 28461 (2000).

[29] R. J. Harrison and J. M. Feinberg, Geochem. Geophys. Geosyst. 9, Q05016 (2008).

[30] J. E. Davies, O. Hellwig, E. E. Fullerton, J. S. Jiang, S. D. Bader, G. T. Zimányi, and K. Liu, Appl. Phys. Lett. 86, 262503 (2005).

[31] F. Preisach, Z. Phys. 94, 277 (1935).

[32] I. D. Mayergoyz, Phys. Rev. Lett. 56, 1518 (1986).

[33] C.-I. Dobrotă and A. Stancu, J. Appl. Phys. 113, 043928 (2013).
[34] C.-I. Dobrotă and A. Stancu, Phys. B (Amsterdam) 457, 280 (2015).

[35] J. Gräfe, F. Haering, T. Tietze, P. Audehm, M. Weigand, U. Wiedwald, P. Ziemann, P. Gawroński, G. Schütz, and E. J. Goering, Nanotechnology 26, 225203 (2015).

[36] J. Gräfe, M. Schmidt, P. Audehm, G. Schütz, and E. Goering, Rev. Sci. Instrum. 85, 023901 (2014).

[37] D. Nolle, M. Weigand, P. Audehm, E. Goering, U. Wiesemann, C. Wolter, E. Nolle, and G. Schütz, Rev. Sci. Instrum. 83, 046112 (2012).

[38] W. Rasband, ImageJ, U.S. National Institutes of Health, Bethesda, Maryland, USA, http://imagej.nih.gov/ij/, 1997-2015.

[39] P. Thevenaz, U. E. Ruttimann, and M. Unser, IEEE Trans. Image Process. 7, 27 (1998).

[40] See Supplemental Material at http://link.aps.org/supplemental/ 10.1103/PhysRevB.93.014406 for a video of the full magnetization reversal including additional field steps. 